[0212-7199 (2004) 21: 2; pp 75-78] ANALES DE MEDICINA INTERNA Copyright @ 2004 ARAN EDICIONES, S.L. AN. MED. INTERNA (Madrid) Vol. 21, N. $^{\circ} 2$, pp. $75-78,2004$

\title{
Listeriosis en el adulto. Revisión de 10 casos
}

\author{
I. M. ARIAS MIRANDA, F. J. NUÑO MATEO, J. NOVAL MENÉNDEZ, \\ E. M. FONSECA AIZPURU, M. J. MENÉNDEZ CALDERÓN
}

Servicio de Medicina Interna. Hospital de Cabueñes. Gijón

LISTERIOSIS IN THE ADULT. REVISION OF 10 CASES

\section{RESUMEN}

La Listeria monocytogenes (LM) continúa siendo una rara infección oportunista en enfermos inmunodeprimidos. Se presentan las características clinicoepidemiológicas y terapéuticas de 10 casos de infección por LM, siendo estas 4 bacteriemias primarias, 3 meningitis, 2 peritonitis bacterianas espontáneas y 1 absceso abdominal. Todos tenían factores predisponentes. Las formas de presentación más frecuentes fueron el cuadro séptico y la meningoencefalitis. El antibiótico más utilizado fue la ampicilina. La mortalidad global fue de un $40 \%$.

PALABRAS CLAVE: Listeria monocytogenes. Infección. Epidemiología.

\begin{abstract}
Listeria monocytogenes is still a very rare opportunist infection in immunosuppressive patients. The clinical-epidemiological and therapeutic characteristics in 10 patients with infection produced by LM are reported, - four of them had primary bacteriemia, three patients had a meningeal involvement, there were two patients with spontaneous bacterial peritonitis and one suffered from abdominal access. All of the patients had underlying disorders favouring the infection. Sepsis and meningeal syndrome were the most common presenting forms. Ampicilin was the most used antibiotic. The overall mortality was $40 \%$.
\end{abstract}

KEY WORDS. Listeria monocytogenes. Infection. Epidemiology.

Arias Miranda IM, Nuño Mateo FJ, Noval Menéndez J, Fonseca Aizpuru EM. Menéndez Calderón MJ. Listeriosis en el adulto. Revisión de 10 casos. An Med Interna (Madrid) 2004; 21: 75-78.

\section{INTRODUCCIÓN}

Listeria monocytogenes (LM) es un bacilo gram positivo asociado a infecciones en mujeres embarazadas y responsable de sepsis neonatal. En adultos no gestantes es una infrecuente pero severa infección que usualmente afecta a pacientes inmunodeprimidos.

En este trabajo describimos nuestra experiencia en listeriosis del adulto resaltando factores predisponentes, curso clínico, respuesta terapéutica y pronóstico en una serie de 10 casos.

\section{CASOS APORTADOS}

Presentamos un estudio retrospectivo efectuado en el hospital de Cabueñes (grupo 2 del antiguo Insalud ahora SESPA) que atiende a una población cercana a los 300.000 habitantes en Gijón, Principado de Asturias, y que carece de los servicios de neurocirugía, cirugía vascular y maxilofacial, y en el que no se realizan transplantes de órganos. Nosotros revisamos las historias de todos los pacientes con cultivo positivo para LM entre enero de 1991 y diciembre de 2002. Se consideró caso de listeriosis del adulto al episodio de enfermedad compatible en paciente con aislamiento de LM en localización habitualmente estéril, en mayores de 14 años. Se definió como sepsis primaria cuando los hemocultivos fueron positivos, en ausencia de infección en otros lugares del organismo. Se diagnosticó de meningitis o meningoencefalitis al hallazgo de LM en líquido cefalorraquídeo (LCR), y en los casos con cultivo negativo del LCR en los que se observaron signos citoquímicos de inflamación meníngea siempre que los hemocultivos fueran positivos. Se definió caso adquirido en la comunidad aquel cuyo periodo de tiempo entre la fecha de ingreso y la fecha de aislamiento del microorganismo en relación con la toma de la muestra, fuese inferior a 72 horas, mientras que se consideró nosocomial aquel con un periodo superior o igual a 3 días.

Se hallaron 10 casos cuyas principales características se describen en la tabla I. El rango de edad fue de 28 a 81 años con una media

Trabajo aceptado: 29 de agosto de 2003 
de 54 y predominio masculino (80\%). Todos pertenecían a la comunidad del Principado de Asturias. Entre los antecedentes destacaba un hábito etílico mayor de $80 \mathrm{~g}$ de etanol/día en 5 pacientes (50\%), neoplasias sólidas en 3 pacientes (30\%), hemopatía en tratamiento inmunosupresor en 2 pacientes (20\%), cirrosis hepática en 2 pacientes $(20 \%)$, infección por VIH en $20 \%$ ( 2 episodios en el mismo paciente) y enfermedad de Crohn con corticoterapia en 1 paciente (10\%). La adquisición fue comunitaria en el $80 \%$, siendo el $20 \%$ de origen nosocomial. La forma de presentación más frecuente fue como sepsis primaria en 4 pacientes $(40 \%)$ seguido de meningitis en 3 pacientes (30\%) y formas localizadas en 3 casos (30\%), 2 como peritonitis bacteriana espontánea (PBE) y uno como absceso abdominal. Los síntomas clínicos más frecuentes fueron fiebre, que se observó en todos los casos, seguido de síndrome meníngeo en un $30 \%$, diarrea en un $30 \%$ y dolor abdominal en otro $30 \%$. No se halla- ron signos de rombencefalitis, afectación cerebelosa o formación de abscesos cerebrales en las afecciones meníngeas.

En el examen del LCR (Tabla II) se detectó hipoglucorraquia e hiperproteinemia en todos los casos; el recuento celular del LCR mostró una media de 839 células, con predominio polimorfonuclear en 2 casos y linfocitario en uno. El examen del líquido ascítico en los casos de PBE tuvo una media de 4.230 células con predominio polimorfonuclear en uno y linfocitario en el otro.

El aislamiento de LM se realizó en hemocultivos en 8 casos, de forma conjunta en sangre y LCR en los casos de la forma meníngea y en los 2 casos de PBE únicamente en líquido ascítico.

En el tratamiento la ampicilina se utilizó en 8 casos con dosis adecuadas y asociado generalmente a aminoglucósidos y cefalosporinas de tercera generación. En un paciente alérgico a la penicilina y derivados se utilizó cotrimoxazol con respuesta clínica inicial ade-

TABLA I

CARACTERÍSTICAS DE 10 EPISODIOS DE LISTERIOSIS EN ADULTO

\begin{tabular}{llllll}
\hline Caso Edad/sexo & Fecha & $\begin{array}{l}\text { Factores } \\
\text { predisponentes }\end{array}$ & Presentación & $\begin{array}{l}\text { Aislamiento de } \\
\text { Listeria monocytogenes }\end{array}$ & Tratamiento \\
\hline 1 & $30 / \mathrm{M}$ & Sep 1990 & $\mathrm{VIH}$, etilismo & Síndrome meníngeo y fiebre & $\begin{array}{l}\text { Sangre y LCR } \\
\begin{array}{c}\text { Cloxacilina, gentamicina, } \\
\text { vancomicina, ampicilina } \\
\text { y eritromicina }\end{array}\end{array}$
\end{tabular}

$2 \quad 31 / M \quad S e p 1991 \quad$ VIH, etilismo

3

4

5

53/V Nov 2001

E

$\begin{array}{ccc}\text { 40/V Ago } 2002 \quad \begin{array}{l}\text { Enf. Hodgkin (IIIB) } \\ \text { y QT }\end{array} & \end{array}$

10

81/V Sep 2002 intervenido

COD

Linfoepitelioma de cavum, QT y RT

Fiebre, diarrea

Síndrome meníngeo y fiebre Sangre y LCR

Fiebre nosocomial

pancitopenia y RT

Cirrosis Child C, diabetes, etilismo

Dolor abdominal, fiebre y ascitis

Cirrosis Child C, VHC+, Diarrea, ascitis, fiebre etilismo y deterioro general

Enf. Crohn, corticoterapia

Dolor abdominal, fiebre y plastrón abdominal

Sindrome meníngeo, fiebre y letargia

Sd febril

Deterioro general y fiebre nosocomial
Sangre

Sangre

Sangre y LCR

Ampicilina y cefotaxima

Favorable

Ampicilina y cefotaxima

Exitus

Sangre

Líquido ascítico

Líquido ascítico

Cefotaxima y ampicilina

Exitus

Piperacilina-tazobactan,

Cefotaxima y ampicilina

Favorable

Exitus

(2)

Cirugía, ampicilina y metronidazol

Favorable

Cotrimoxazol, imipenem, meropenem, cefotaxima y rifampicina

Ampicilina y gentamicina Favorable

Sangre

Sangre

Ciprofloxacino

Exitus $\overline{\mathrm{VIH}}$ : virus de la inmunodeficiencia adquirida. QT: quimioterapia. RT: radioterapia. VHC: virus de la hepatitis C. COD: cáncer de origen desconocido.

\section{TABLA II}

CARACTERÍSTICAS DEL LCR EN LOS CASOS DE MENINGITIS

\begin{tabular}{lcccccc}
\hline Caso & Células & PMN (\%) & Glucosa & Proteínas & LCR & Hemocultivos \\
\hline 1 & 213 & Predominio linfocitario & $17 \mathrm{mg} / \mathrm{dl}$ & $0,8 \mathrm{~g} / \mathrm{l}$ & + & + \\
3 & 806 & $90 \%$ & $9 \mathrm{mg} / \mathrm{dl}$ & $0,66 \mathrm{~g} / \mathrm{l}$ & + & + \\
8 & 1500 & $91 \%$ & $27 \mathrm{mg} / \mathrm{dl}$ & $5,1 \mathrm{~g} / \mathrm{l}$ & + & + \\
\hline
\end{tabular}


MASTER Tratamiento del Dolor

Online/Presencial

\section{UNIVERSIDAD DE SALAMANCA}

\section{$\mathbf{5 0 0}$ horas lectivas}

Dirigido a:

Profesionales de todos los ámbitos de la Medicina (clínica, de familia, anestesiología, traumatología, pediatría, reumatología, oncología, c. paliativos, cirugía, etc.), Farmacia, Psicologia, e Industria Farmacéutica

\section{CURSO EXTRAORDINARIO 75 horas}

"Planteamientos Básicos en el Tratamiento del Dolor" 250 hioras $s+100 \mathrm{~h}$. proyecto de investigación) EXPERTO UNIVERSITARIO Experto Universitario en el Tratamiento del Dolor

\section{MÁSTER}

Máster en el Tratamiento delDolor

Módulios especificos según especialidad

Prácticas en Unidades de Dolor y Laboratorios de Investigación Clinica

Dirección Académica: Prof. Clemente Muriel Villoria

Catedrático de Anestesiología, Reanimación y Terapia del Dolor Jefe del Servicio de Anestesia y Reanimación del Hospital Clínico Universitario de Salamanca 
cuada, pero con posterior retirada por desarrollo de una acidosis láctica secundaria, completando el tratamiento con imipenem con respuesta satisfactoria. Un paciente al que se le trató con ciprofloxacino falleció en las primeras 24 horas de iniciada la sintomatología.

La mortalidad fue de un $40 \%$, todos ellos portadores de neoplasia avanzada o cirrosis en estadio final. No se hallaron secuelas en los pacientes que se recuperaron. Durante el seguimiento al resto de los pacientes fallecieron en los 6 meses siguientes por progresión de la enfermedad de base, sólo superviviendo el paciente con enfermedad de Crohn.

\section{DISCUSIÓN}

Listeria monocytogenes es el principal patógeno humano del género listeria, distribuído ampliamente en la naturaleza y transmitido fundamentalmente por la ingesta de alimentos contaminados. Se han descrito diversos episodios epidémicos reconociéndose el alimento causante y su mecanismo de contaminación $(1,2)$.

En adultos sanos su exposición suele provocar un corto periodo de portador asintomático con eliminación en heces (3); sin embargo en mujeres embarazadas, neonatos, ancianos y adultos con alteraciones inmunitarias puede llevar a un cuadro de listeriosis invasiva (4).

La baja incidencia de listeriosis encontrada en nuestra revisión se basa en la baja capacidad de este microorganismo de producir enfermedad en el ser humano y por el hecho de presentarse como casos esporádicos sin detectarse ningún brote epidémico. En España, concretamente en el área de Barcelona, se realizó un estudio objetivando una tasa de incidencia global de 9,4 casos por millón de habitantes/año (5).

Coincidiendo con otros estudios publicados, existe un predominio en varones (6-8) y un cierto predominio de la infección en humanos entre los meses de julio a octubre (9).

La listeriosis aunque puede afectar a población sana, es una infección que incide en pacientes afectos de enfermedad previa y/o tratamiento inmunosupresor (10-12). La totalidad de nuestra serie presentaba factores predisponentes destacando por orden de frecuencia etilismo crónico, neoplasias sólidas y hematológicas en tratamiento inmunosupresor, cirrosis hepática, infección por VIH y enfermedad de Crohn. No encontramos ningún caso en mujer embarazada.

Habitualmente la listeriosis es adquirida en la comunidad, no obstante cada vez hay más casos descritos de origen nosocomial $(13,14)$. Esto se explica siendo el paciente al ingreso portador crónico y posteriormente durante la hospitalización ya sea por malnutrición, progresión de la enfermedad o tratamiento inmunosupresor se provocaría el desarrollo de la enfermedad. Presentamos 2 casos (casos 3 y 10) con estas últimas características, con estancia superior al mes e infección invasiva y fulminante, llevando al fallecimiento del paciente en pocas horas.

Las formas clínicas de presentación más frecuentes fueron la sepsis primaria y la meningitis con características similares a otras series (15-19); destacamos sin embargo un 30\% de formas localizadas.

La asociación HIV- listeriosis es excepcional, así en España sólo hemos encontrado 6 publicaciones $(7,20-24)$ y se relaciona sobre todo con el uso de cotrimoxazol para la prevención de infección oportunista. Nuestra paciente con infección por VIH (casos 1 y 2) debutó con una infección invasiva por LM con posterior reinfección al cabo de un año con forma clí- nica diferente, sin constatarse antecedente de tratamiento con cotrimoxazol profiláctico y permaneciendo con niveles de CD4>200 en los 2 episodios.

La peritonitis bacteriana espontánea (PBE) por LM es un cuadro poco frecuente estando descritos unos 30 casos en el mundo $(25,26)$. Los pacientes con cirrosis hepática tienen anomalías en los mecanismos de defensa, incluyendo la disfunción en el sistema reticuloendotelial, reducción en los niveles de complemento y disminución en la actividad de los neutrófilos y macrófagos considerándose por todo ello grupo de riesgo para el desarrollo de listeriosis $(27,28)$. La mayoría de los casos están descritos en pacientes cirróticos en España, atribuyéndose a hábitos dietéticos específicos y predisposición geográfica (29-31). Presentamos 2 nuevos casos (casos 5 y 6) en cirróticos de origen etílico y VHC en estadio C de Child- Pugh con peritonitis como única localización. La cefotaxima, antibiótico empírico de elección en las PBE, no sería eficaz por lo que destacamos la importancia de una comunicación temprana por parte del servicio de Microbiología ante la mínima sospecha para conseguir una adecuada cobertura.

La enfermedad de Crohn y su tratamiento (32) son condiciones predisponentes para la inmunodepresión del paciente. Los abscesos abdominopélvicos en la enfermedad de Crohn ocurren en un $20 \%$, siendo importante valorar esta complicación ya que la corticoterapia a dosis altas puede agravar la situación. El aislamiento de LM en cuadros de afectación local como el incluído en nuestra serie es infrecuente, siendo los bacilos gram negativos los principales causantes de esta patología (33). El tratamiento sería la antibioterapia adecuada acompañada de la cirugía, en los casos que requiriesen drenaje $(34,35)$. A propósito de este caso, destacar según múltiples estudios el papel de la LM en la patogenia de la enfermedad de Crohn; agentes infecciosos como E. coli, streptococos, LM y virus han sido estudiados para demostrar su contribución en el origen de esta enfermedad (36-39).

La LM es sensible in vitro a un grupo de antibióticos incluído penicilina $\mathrm{G}$, eritromicina, ampicilina, cotrimoxazol, cloranfenicol, tetraciclina y aminoglucósidos; actualmente se tiende a utilizar ampicilina sola o asociada a aminoglucósidos y cotrimoxazol en los casos de alergia a penicilina y derivados $(15,40,41)$. En nuestra serie el tratamiento fue adecuado en la mayoría de los casos tras la información por parte del servicio de Microbiología de su aislamiento, si exceptuamos el caso de un paciente (caso 10) que falleció tras un cuadro séptico con resultado microbiológico posterior a su fallecimiento. No hubo casos de resistencia a penicilina y sí una reacción adversa a cotrimoxazol en un paciente alérgico a penicilinas que desarrolló una acidosis láctica.

La mortalidad en distintas series oscila habiéndose descrito desde el $28 \%$ hasta el $40 \%$ (42). En nuestra serie alcanzó el $40 \%$ siendo el factor crítico el deterioro severo inducido por la enfermedad subyacente. No se produjo ninguna secuela ni recaída en los pacientes que superaron la infección.

En resumen la listeriosis permanece como una grave y rara infección oportunista. En nuestro medio consideramos que la incidencia aumentará en la edad adulta, relacionado con el progresivo envejecimiento de la población y por el incremento y mayor esperanza de vida de los pacientes inmunodeprimidos. La realización en la población susceptible de medidas de profilaxis adecuadas y la sospecha clínica en los casos de la enfermedad junto a un diagnóstico y tratamiento antibiótico temprano, serían las medidas para evitar su alta mortalidad. 


\section{Bibliografía}

1. Outbreak of Listeriosis- Northeastern United States, 2002. MMWR 2002; 51: 950-951.

2. Outbreak of Listeriosis Associated With Homenade Mexican-Style Cheese-North Carolina, October 2000-January 2001. MMRW 2001; 50: 560-562.

3. Grif K, Patscheider G, Dierich MP, Allerberger F. Incidence of Fecal Carriage of Listeria monocytogenes in Three Healthy Volunteers: A One-Year Prospective Stool Survey. Eur J Clin Microbiol Infect Dis 2003; 22: 16-20.

4. Eleftherios Mylonakis, Maria Palou, Elisabeth L. Hohmann, Stephen B Calderwood, Edward J. Wing. Listeriosis During Pregnancy. A Case Series and Review of 222 Cases. Medicine 2002; 81: 260-269.

5. Nolla-Salas J, Plasencia A, Gasser I, Almela M, Coll P, Antó JM y Grupo de Estudio de la Listeriosis en Barcelona. Estudio clinicoepidemiológico de la listeriosis humana en Barcelona (1990-1991). Med Clin (Barc) 1994; 103: 41-45.

6. Eleftherios Mylonakis, Elisabeth L. Hohmann, stephen B. Calderwood. Central Nervous Sistem Infection with Listeria monocytogenes. 33 Years'Experience at a General Hospital and Review of 776 Episodes from tha Literature. Medicine 1998; 77: 313-336.

7. Alcoba Lez M, Carro Fernández JA, Pérez Simón MR, Guerra Laso J, Alonso Fernández A, Arto Millán L. Meningitis por Listeria monocytogenes en el adulto en España. Presentación de 10 casos y revisión de la literatura. Rev Clin Esp 2002; 202 (12): 638-643.

8. Elcuaz R, Bordes A, Aladro Y, García A, Perera A, Valle L, Cañas F, Lafarga B. Características clínicas y estudio epidemiológico de un brote de listeriosis en Gran Canaria. Enferm Infecc Microbiol Clin 1996; 14: 416-421.

9. Einar KR. Hjaltested, Sigrún Gudmundsdóttir, Kristín Jónsdóttir, Karl G. Kristinsson, Ólafur Steingrímsson, Már Kristjánsson. Listeriosis in Iceland, 1978-2000: A Description of cases and Molecular Epidemiology. Scand J Infect Dis 2002; 34: 734-741.

10. Ruiz Pérez FJ, Méndez Martínez P, Ortiz Romero MM, Martínez Garcerán JJ, Bolarín López J, Sánchez Gascón F. Neumonía por Listeria monocytogenes: una severa complicación del paciente neoplásico. An Med Interna (Madrid) 1999; 16: 420-422.

11. Girmenia C, Iori AP, Testi AM, Moleti ML, Arcese W, Martino P. Listeriosis in Recipients of Allogeneic Bone Marrow Transplants from Unrelated Donors. Eur J ClinMicrobiol Infect Dis 2000; 19: 711-714.

12. Martín Aragonés G, Sanz MA, Martínez JA, Rafecas FJ, Sempere A, Pérez-Bellés, Gobernado M. Infección por Listeria monocytogenes en pacientes leucémicos. A propósito de cinco observaciones. Enf Infec Microbiol Clin 1988; 6(8) : 367-371.

13. Stamm AM, Dismukes WE, Simmons BP et al. Listeriosis in renal transplant recipients: report of an ourbreak and review of 102 cases. Rev Infect Dis 1982; 4: 665-682.

14. Moore RM, Zehmer RB, Listeriosis in the United States 1971. J Infect Dis 1973; 127: 610-611.

15. Julián A, Jiménez A, de Górgolas M, Fernández R, Fernández ML. Infecciones por Listeria monocytogenes en el adulto Aspectos clínicos y microbiológicos de una enfermedad cambiante. Enferm Infecc Microbiol Clin 2001; 19: 297-303.

16. Hernández A, Almirante B, Pahissa A, Capdevila JA, Planes AM, Gasser I, Martínez Vázquez JM. Listeriosis en el adulto. Consideraciones clínicas epidemiológicas y terapéuticas basadas en una serie de 26 casos. Enf Infec y Microbiol Clin 1990; 7: 414-419.

17. Büla CJ, Bille J, Glauser MP. An Epidemic of Food-Borne Listeriosis in Western Switzerland : Description of 57 Cases Involving Adults. Clin Infect Dis $1995 ; 20: 66-72$.

18. Aguado García JM, Fernández Guerrero ML, Gómez Garces JL, Soriano García F, Ales Reinlein JM. Infecciones por Listeria monocytogenes en el adulto. Experiencia en la última década 1971-1981. Rev Clin Esp 1983 (171); 2: 93-98.

19. Domingo Pedrol P, Mancebo Cortés J, Blanch Torra L1, Coll Figa P, Net Castel A, Nolla Panadés J. Meningitis por Listeria monocytogenes en adultos. Estudio de 10 casos. Rev Clin Esp 1986; 178 (4): 158-163.

20. Valencia Ortega ME, Enríquez Crego A, Laguna Cuesta F, Ortega Millán G, González Lahoz JM. Listeriosis. Una infección poco fre- cuente en pacientes con VIH. An Med Interna (Madrid) 2000; 17: 649651.

21. García Castaño J, Farfán Sedano Al, Jirones Pérez JM, Ortiz Vega M. Meningitis por Listeria monocytogenes tras colonoscopia en paciente con infección por VIH y citomegalovirus. An Med Interna (Madrid) 2001; 18: 398-9.

22. Roldán A, Gutiérrez A, Jiménez J, Agulla A, Zancada F, Fernández A. Meningitis por Listeria e infección por VIH. Rev Clin Esp 1991; 188: $187-8$

23. Berenguer J, Solera J, Díaz MD, Moreno S, López- Herce JA, Bouza E. Listeriosis in patients infected with human inmunodeficiency virus. Rev Infect Dis 1991; 13: 115-9.

24. Sánchez B, Yebra M, Lacoma F, Gea JC. Meningitis por Listeria monocytogenes en un paciente infectado por el virus de la inmunodeficiencia humana. Enf Infec Microbiol Clin 1991; 9: 386-7.

25. Nolla-Salas J, Almera M, Gasser I, Latorre C, Salvadó M, Coll P. Spontaneus Listeria monocytogenes Peritonitis. A population-Based Study of 13 Cases Collected in Spain. Am J Gastroenterol 2002; 97: 15071511.

26. Uriarte Retuerto I, Bujanda Fernández de Piérola L, Peña Sarnago P. Peritonitis bacteriana espontánea por Listeria monocytogenes.An Med Interna (Madrid) 2002; 19: 214-215.

27. Amador C, Bañares R, Clemente G, Plasquau F, Guiraldo A. Peritonitis bacteriana espontánea por Listeria monocytogenes. Enf Infect Microbiol Clin 1988; 6: 361-373.

28. Jayaraj K, Di Bisceglie AM, Gibson S. Spontaneous bacterial peritonitis caused by infection with Listeria monocytogenes: A case report and review of the literature. Am J Gastroenterol 1998; 93: 1556-8.

29. Adeonighbagbe O, Khadami A, Karowe M, Gualtieri N, Robilotti J. Listeria moncytogenes peritonitis : an unusual presentation and review of the literature. Clin Gastroenterol 2000; 30 (4): 436-7.

30. Jensen A, Frederiksen W, Gerner-Smidt P. Risk factors for listeriosis in Denmark 1989-1990. Scand J Infect Dis 1994; 26: 171-8.

31. Manso C, Rivas I, Peraire J, et al. Fatal Listeria meningitis, endocarditis and pericarditis in a patient with hemochromatosis. Scand J Infect Dis 1997; 29: 308-9.

32. Slifman NR, Gershon SK, Lee JH, Edwards ET, Braun MM. Listeria monocytogenes Infection as a Complication of Treatment With Tumor Necrosis Factor a-Neutralizing Agents. Artrithis Rheum 2003; 48 (2): 319-324.

33. Sile H, Norwood J. Intra-abdominal abscess caused by Listeria monocytogenes in a patient with acqute hemolytic anemia and trombocytopenia. South Med J 2002; 95 (11): 1350-2.

34. Jawari A, Kamm MA, Ong C, Forbes A, Bartram CL, Hawley PR. Intra- abdominal and pelvic abscess in Crohn's disease: results of non invasive and surgical management. Br J Surg 1998; 85: 367-371.

35. Chiba M, Fukushima T, Inoue S, Horie Y, lizuka M, Masamune O. Listeria monocytogenes in Crohn's disease. Scand J Gastroenterol 1998; 33 (4): 430-4.

36. Brown WR. Listeria: the latest putative pathogenetic microorganism in Crohn's disease. Gastroenterology 1995; 108 (5): 1589-90.

37. Liu Y, van Kruiningen HG, West AB, Cartun RW, Cortot A, Colombel JF. Inmunocytochemical evidence of Listeria, Escherichia coli and Streptococcus antigens in Crohn's disease. Gastroenterology 1995; 108 (5): 1396-404.

38. Blaser MJ, Miller RA, Lacher J, Singleton JW. Patients with active Crohn's disease have elevated serum antibodies to antigens of seven enteric bacterial pathogens. Gastroenterology 1984; 87 (4): 888-94.

39. Andus T, Gross V. Etiology and pathophisiology of inflammatory bowel disease- environmental factors. Hepatogastroenterology 2000; 47 (31): 29-43.

40. Khayr WF, Cherubin CE, Bleck TP. Listeriosis a protean disease. Infect Dis Clin Pract 1992; 1: 291-298.

41. Hof H, Nichterlein T, Kretschmar M. Management of listeriosis. Clin Microbiol Rev 1997; 39: 345-357.

42. Humbert G, Duval CL, Fessard CL, et al. Aspects actuels des listerioses en France (a propos d'une statistique de 824 cas). Ire parti. Lyon Med 1977; 4: 275-289. 